\title{
Rain Fade Mitigation Technique Using Residue Number System Architecture on KU Band Satellite Communication Link
}

\author{
Stephen Akobre ${ }^{1, *}$, Mohammed Ibrahim Daabo ${ }^{2}$, Abdul-Mumin Salifu ${ }^{3}$ \\ Faculty of Computing and Information Sciences, C. K. Tedam University of Technology and Applied Sciences, Navrongo, Ghana
}

Email address:

sakobre@cktutas.edu.gh (S. Akobre), i.daabo@cktutas.edu.gh (M. I. Daabo), asalifu@cktutas.edu.gh (A. M. Salifu)

${ }^{*}$ Corresponding author

\section{To cite this article:}

Stephen Akobre, Mohammed Ibrahim Daabo, Abdul-Mumin Salifu. Rain Fade Mitigation Technique Using Residue Number System Architecture on KU Band Satellite Communication Link. Advances in Networks. Vol. 8, No. 1, 2020, pp. 1-8.

doi: $10.11648 /$ j.net.20200801.11

Received: August 26, 2020; Accepted: October 13, 2020; Published: October 20, 2020

\begin{abstract}
Rain fade is the loss of signal power at the receiver of a telecommunication system mainly due to absorption and scattering caused by rain in the transmission medium, especially at frequencies above $10 \mathrm{GHz}$. In order to combat the loss of the signal power at the receiver, there is the need to employ rain fade mitigation techniques. Consequently, researchers have been studying how rain affects the signal in different geographical locations as well as proposing some mitigation techniques. Power control is one of the mitigation techniques that have been proposed. But this technique has some associated challenges. Increasing the power will lead to an increase in cost of transmission which will eventually be passed on to the consumer thereby making satellite services expensive. It introduces a delay in compensation due to link estimation and coordination. Also, because of health concerns there is a limit to the amount of power that can be radiated to the ground and this is governed by international agreements. Another power management drawback in using this technique is that, it is essential to track the power continuously to ensure that the power values are not set too high, which can lead to the front end of the receiver being overdriven and eventually leading to a shutdown or physical damage. In this paper, we address the power control challenges, by leveraging on the inherent properties of Residue Number System (RNS) to propose an RNS architecture using the moduli set $\left\{2^{2 \mathrm{n}+1}-1,2^{2 \mathrm{n}}-1,2^{2 \mathrm{n}}\right\}$ that can mitigate rain fade in the satellite link. In digital communication systems, the bit energy, $\mathrm{e}_{\mathrm{b}}$, is the most important parameter in determining the communications link performance. Numerical analysis shows that the proposed scheme performs better than the traditional method as indicated in the high energy per bit value obtained in the proposed system in comparison with the traditional method.
\end{abstract}

Keywords: Rain Fade Mitigation, Power Control, Residue Number System

\section{Introduction}

The immense strength of satellite broadcasting lies in its ability to access a limitless number of sites without the need for any physical links irrespective of their location. A satellite receives the up-linked signal, lowers its frequency and rebroadcasts it to any geographical area desired. Ku band satellites are designed for spot beams, operating in the higher range of $12 \mathrm{GHz}$ and allow smaller antennas to capture their signals [1]. The satellite signal transmission process is shown in Figure 1.

Rainfall is known to be the major cause of signal impairment in Ku band. In view of this, researchers have been developing rain rate and rain attenuation models to characterise and predict the effects of rain and propose mitigation techniques. However, very little has been done with regards to rain attenuation mitigation. A research was conducted on mitigation technique for rain fade using frequency diversity method by [2] in Malaysia. The frequency diversity method was used; however, this technique is not suitable because ground stations and satellites using this technique must be equipped to operate in dual frequency mode. The method is also complex because the receiver will have to pick up all the different signals. Power control is one of the mitigation techniques that have been 
proposed. In 2019, [3] conducted a research on rain attenuation mitigation on wireless communication link using adaptive power control. The model employed a feedback-based transmission power control algorithm to dynamically maintain individual link quality over time using Proportional Integral Derivative (PID). They concluded that PID has an enhanced response because it has shorter rising time and setting time when compared with proportional and proportional Integral controller systems. Their solution also focused on $5 \mathrm{GHz}$ spectrum. But the use of power control is ineffective and expensive. This is because a satellite transmitter that offers coverage to a variety of users at different geographic location needs to work continuously at or near its peak power to overcome the overall attenuation encountered by only one of the ground stations. Again there are concerns regarding the safety of the amount of power that can be radiated to the ground and this is governed by international agreements [4]. Moreover, there are issues of intersystem interference with the increase in power level. This technique also requires some form of user intervention. Therefore it is imperative that other methods be considered to combat the effect of rain fade and restore acceptable performance on the communication link other than increasing the power. In this research, we leverage on the inherent properties of RNS in developing a cost effective solution to mitigate the effect of rain attenuation on the satellite communication link. This is achieved by using converters that changes the number system to RNS architecture before transmission. This is done using a forward converter at the transmitter. A reverse converter at the receiver then converts back from RNS to the traditional number representation. By this procedure, the energy per bit can be greatly enhanced so that even in the unlikely event of the signal encountering a rain event on the link, there will still be sufficient energy at the receiver to allow for the proper decoding of data.

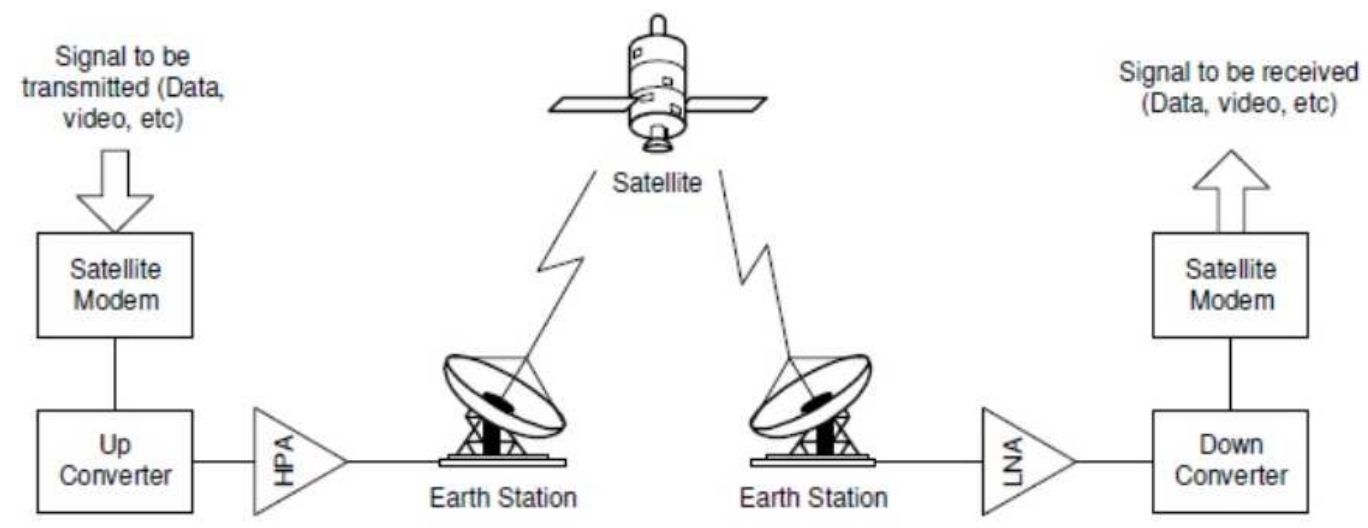

Figure 1. The Satellite Transmission System.

\section{Residue Number System}

Residue Number System (RNS) is a number system with numerous advantages. It is a well-established fact, that a number of digital devices naturally depend on number systems and to a large extend digital devices are built using binary number system. RNS has been used efficiently in communication systems [5], Digital Signal Processing for filtering, Convolutions and Correlations [6]. Residue Number System (RNS) is defined by the set $\mathrm{S}$ which includes $\mathrm{N}$ integers that are pair-wise relatively prime. That is $\mathrm{S}=\left\{m_{1}, m_{2}, \ldots m_{N}\right\}$, where $\operatorname{gcd}\left(m_{i}, m_{j}\right)=1$ for $i, j=1, \ldots N$ and $i, j \neq 1$ and ged means the greatest common divisor [7]

Every integer $\mathrm{X}$ in $[0, M-1]$ can be uniquely represented with an $\mathrm{N}$-tuple where,

$$
\begin{gathered}
M=\prod_{i=1}^{n} m_{i}, X \rightarrow\left(R_{1}, R_{2}, \ldots \ldots \ldots R_{N}\right) \text { and } R_{i}=|X|_{m_{i}}= \\
\left(X \bmod m_{i}\right) ; \text { for } i=1 \text { to } N .
\end{gathered}
$$

The set $\mathrm{S}$ and the number $R_{i}$ are called the moduli set and the residue of $X$ modulo $m_{i}$, respectively [8-10].

Over the past years there has been renewed interest especially in the area of arithmetic computation and signal processing applications such as Fast Fourier transforms, digital filtering and image processing [11-14]. The inherent carry free operations, parallelism, borrow-free subtraction, single step multiplication without partial product and fault-tolerance properties of Residue Number System have made it a choice of technology for high precision and high throughput rate Digital Signal Processing applications where only repeated multiplications and additions are required [15-17].

\section{The Proposed Design}

The complexity and varied problems associated with rain attenuation mitigation techniques makes it necessary to explore other methods. One way is the reduction in the data rate. But instead of reducing the size of data to be transmitted or transmitting one piece at a time, a better approach to use is by employing a different number system. The RNS is the most reliable solution, since this number system is able to reduce a given decimal to residues in respect to a given moduli set. And thus the residues to be transmitted will amount to fewer bits than the number of bits produced by the conventional number system. Therefore designing converters that can greatly reduce the number of bits before transmission will result in a higher energy-per-bit to noise density ratio which will lead to a higher carrier-to-noise ratio at the receiver leading to better performance of the communication link. Another advantage is that the system does not require human intervention during 
rain attenuation mitigation. The proposed system architecture is shown in Figure 2.

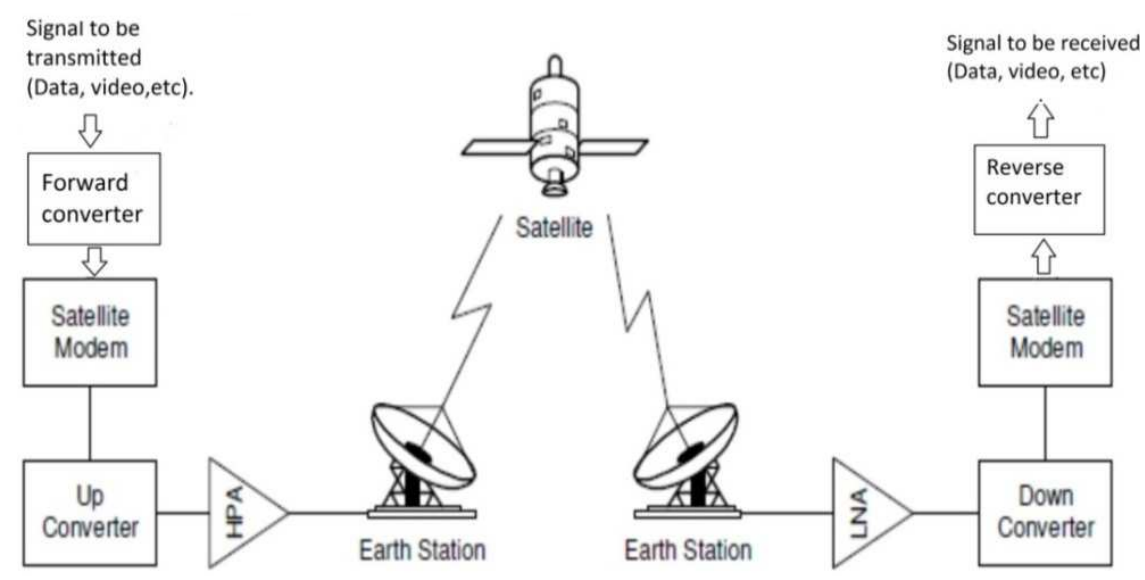

Figure 2. Satellite communication link with RNS converters.

A. Forward Conversion Process For Moduli Set $\left\{2^{2 n+1}-\right.$ $\left.1,2^{2 n}-1,2^{2 n}\right\}$

This moduli set was chosen because it gives a large dynamic range for small values of $\mathrm{n}$. In satellite communication it is advisable to transmit large amount of data at a time because of the long delay. The moduli set of the proposed scheme works for both odd and even numbers of $n>1$.

Given the moduli set, let

$$
m_{1}=2^{2 n+1}-1, m_{2}=2^{2 n}-1 \text { and } m_{3}=2^{2 n} ;
$$

An integer $X$ in the range $[0, M)$ is a $(6 n+1)$-bit number which binary representation is as follows:

$$
X=X_{6 n} X_{6 n-} \ldots X_{1} X_{0}
$$

This weighted representation has a unique equivalent RNS representation $x_{i}=|X|_{m_{i}} \Leftrightarrow\left(x_{1}, x_{2}, x_{3}\right)$; and in order to compute the $x_{i}$ 's, Equation (1) is partitioned into two $2 n$-bit blocks and a $(2 n+1)$-bit block as:

$$
\left.\begin{array}{c}
\Lambda_{1}=\sum_{j=0}^{2 n-1} x_{j} 2^{j} \\
\Lambda_{2}=\sum_{j=2 n}^{4 n-1} x_{j} 2^{j-2 n} \\
\Lambda_{3}=\sum_{j=4 n}^{6 n} x_{j} 2^{j-4 n}
\end{array}\right\}
$$

this implies

$$
X=\Lambda_{1}+2^{2 n} \Lambda_{2}+2^{4 n} \Lambda_{3}
$$

Such that,

$$
\begin{gathered}
x_{1}=|X|_{2^{2 n+1}-1} \\
=\left|\Lambda_{1}+2^{2 n} \Lambda_{2}+2^{4 n} \Lambda_{3}\right|_{2^{2 n+1}-1} \\
=\left.|| \Lambda_{1}\right|_{2^{2 n+1}-1}+\left|2^{2 n} \Lambda_{2}\right|_{2^{2 n+1}-1}+\left.\left|2^{4 n} \Lambda_{3}\right|_{2^{2 n+1}-1}\right|_{2^{2 n+1}-1} \\
=\left|\Lambda_{1}+2^{2 n} \Lambda_{2}+2^{2 n-1} \Lambda_{3}\right|_{2^{2 n+1}} \\
x_{2}=|X|_{2^{2 n}-1}=\left|\Lambda_{1}+2^{2 n} \Lambda_{2}+2^{4 n} \Lambda_{3}\right|_{2^{2 n}-1} \\
=\left.|| \Lambda_{1}\right|_{2^{2 n}-1}+\left|2^{2 n} \Lambda_{2}\right|_{2^{2 n}}+\left.\left|2^{4 n} \Lambda_{3}\right|_{2^{2 n}}\right|_{2^{2 n}-1} \\
=\left|\Lambda_{1}+\Lambda_{2}+\Lambda_{3}\right|_{2^{2 n}}
\end{gathered}
$$

and

$$
x_{3}=|X|_{2^{2 n}}=\Lambda_{1}
$$

The block diagram for the forward conversion is represented in Figure 3; it employs simple adders made up of Carry Save Adders (CSAs) and Carry Propagate Adders (CPAs).

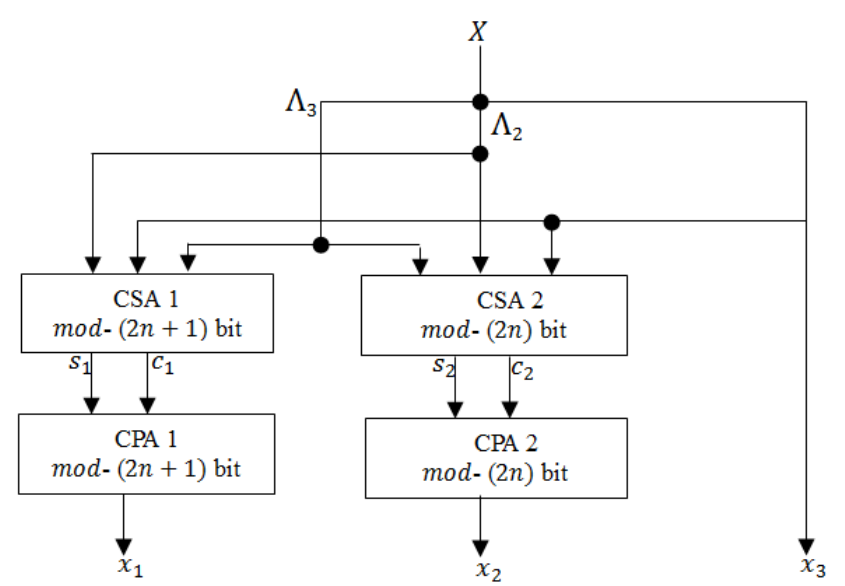

Figure 3. Block Diagram for Forward Convertor. 


\section{B. Bit Energy Computation}

For digital communications, the bit energy, $e_{b}$, is more helpful for determining the link's performance than the carrier power. The link efficiency can be reduced in two ways: if the carrier power, $\mathrm{c}$, is decreased, and/or if the noise power is increased. For example, the carrier power is reduced by the increase in the number of bits to be transmitted and the noise power is increased by the absorption and scattering of the signal by rain drops. Thus the larger the value of $e_{b}$, the better the performance of the link holding the noise power constant. By employing RNS architecture we will greatly reduce the number of bits to be transmitted thereby allowing more power to be available to fewer bits. In Equation (7), a relationship is established between the bit energy and the carrier power as

$$
e_{b}=c T_{b}
$$

where $T_{b}$ is the bit duration in second (s) and $c$ is the carrier power in watts. The energy-per-bit to noise density ratio, $\left(\frac{e_{b}}{n_{o}}\right)$, is the most widely used parameter to evaluate the performance of a digital communication link. And $\left(\frac{e_{b}}{n_{o}}\right)$ is related to $\left(\frac{c}{n_{o}}\right)$ by

$$
X=d_{1}+d_{2} m_{1}+d_{3} m_{1} m_{2}+\cdots+d_{n} m_{1} m_{2} m_{3} \ldots m_{n-1}
$$

Where $d_{i}, i=1,2, \ldots, n$ are the Mixed Radix Digits (MRDs) and computed as follows:

$$
\begin{gathered}
d_{1}=x_{1}, \\
d_{2}=\left.\left.\left|\left(x_{2}-d_{1}\right)\right| m_{1}^{-1}\right|_{m_{2}}\right|_{m_{2}} \\
d_{3}=\left.\left.\left|\left(\left(x_{3}-d_{1}\right)\left|m_{1}^{-1}\right|_{m_{3}}-d_{2}\right)\right| m_{2}^{-1}\right|_{m_{3}}\right|_{m_{3}} \\
\vdots \\
d_{n}=\left.\left.\left|\left(\ldots\left(\left(x_{3}-d_{1}\right)\left|m_{1}^{-1}\right|_{m_{n}}-d_{2}\right)\left|m_{2}^{-1}\right|_{m_{n}}-\cdots-d_{n-1}\right)\right| m_{n-1}^{-1}\right|_{m_{n}}\right|_{m_{n}}
\end{gathered}
$$

Therefore, $X$ in the interval $[0, M)$ can be uniquely represented. And so we can re-write Equation (12) as

$$
\begin{gathered}
d_{1}=x_{1}, \\
d_{2}=\left|\left(x_{2}-d_{1}\right)(1)\right|_{2^{2 n_{-1}}}=\left|x_{2}-x_{1}\right|_{2^{2 n_{-1}}},
\end{gathered}
$$

and

$$
d_{3}=\left|\left(\left(x_{3}-d_{1}\right)(-1)-d_{2}\right)(-1)\right|_{2^{2 n}}=\mid\left(x_{3}-x_{1}+\left.d_{2}\right|_{2^{2 n}}\right.
$$

And Equation (11) then becomes

$$
\begin{aligned}
& X=x_{1}+d_{2}\left(2^{2 n+1}-1\right)+d_{3}\left(2^{2 n+1}-1\right)\left(2^{2 n}-1\right) \\
& =x_{1}+2^{2 n+1} d_{2}-d_{2}+2^{4 n+1} d_{3}-2^{2 n} d_{3}-2^{2 n+1} d_{3}+d_{3}
\end{aligned}
$$

D. Hardware Realization

We now simplify Equations (13) and (14) as follows;

$$
d_{1}=x_{1,2 n} x_{1,2 n-1} \ldots \ldots \ldots x_{1,1} x_{1,0}
$$




$$
\begin{gathered}
d_{2}=\left.\left.|\underbrace{x_{2,2 n-1} x_{2,2 n-2} \ldots \ldots x_{2,1} x_{2,0}}_{2 n-\text { bits }}-| \underbrace{x_{1,2 n-1} \ldots \ldots \ldots x_{1,1} x_{1,0}}_{2 n-2 n}\right|_{2^{2 n}-1}\right|_{2^{2 n}-1} \\
=|\underbrace{x_{2,2 n-1} x_{2,2 n-2} \ldots \ldots x_{2,1} x_{2,0}}_{2 n+1-\text { bits }}+\underbrace{x_{11,2 n-1} x_{11,2 n-2} \ldots \ldots \ldots x_{11,1} x_{11,0}}_{2 n-\text { bits }}|_{2 n-1} \\
=\underbrace{d_{2,2 n-1} d_{2,2 n-2} \ldots \ldots \ldots \ldots d_{2,1} d_{2,0}}_{2 n-\text { bits }}
\end{gathered}
$$

Where, $x_{11}=\left|-x_{1}\right|_{2^{2 n}-1}$ and

$$
\begin{gathered}
d_{3}=|\underbrace{x_{3,2 n-1} \ldots \ldots x_{3,1} x_{3,0}}_{2 n-\text { bits }} \underbrace{x_{11,2 n-1} \ldots x_{11,1} x_{11,0}}_{2 n-\text { bits }}+\underbrace{d_{2,2 n-1} \ldots \ldots d_{2,1} d_{2,0}}_{2 n-\text { bits }}|_{2^{2 n}} \\
=\underbrace{d_{3,2 n-1} d_{3,2 n-2} \ldots \ldots d_{3,1} d_{3,0}}_{2 n-\text { bits }}
\end{gathered}
$$

A simplification of Equation (14) for implementation is as follows:

$$
\begin{aligned}
& X=L_{1}+L_{2}+L_{3}+L_{4} \\
& =\overbrace{0 \ldots 0}^{2 n} \underbrace{L_{1,4 n} \ldots L_{1,1} L_{1,0}}_{4 n+1}+\overbrace{0 \ldots 0}^{2 n} \underbrace{L_{2,4 n} \ldots L_{2,1} L_{2,0}}_{4}+\overbrace{0 \ldots 0}^{2 n+1} \underbrace{L_{3,4 n-1} \ldots L_{3,1} L_{3,0}}_{4 n} \\
& \underbrace{L_{4,6 n} \ldots L_{4,1} L_{4,0}}_{6 n+1}
\end{aligned}
$$

where,

$$
\begin{gathered}
L_{1}=A-d_{2}+d_{3} \\
=\underbrace{A_{4 n} A_{4 n-1} \ldots \ldots A_{1} A_{0}}_{4 n+1}+\overbrace{11 \ldots 1}^{2 n+1} \underbrace{\bar{d}_{2,2 n-1} \ldots \bar{d}_{2,1} \bar{d}_{2,0}}_{2 n-b i t s}+\underbrace{d_{3,2 n-1} \ldots d_{3,1} d_{3,0}}_{2 n-\text { bits }} \overbrace{00 \ldots 0}^{2 n+1}
\end{gathered}
$$

and

$$
\begin{gathered}
A=x_{1}+2^{2 n+1} d_{2} \\
=\underbrace{x_{1,2 n} x_{1,2 n-1} \ldots x_{1,1} x_{1,0}}_{2 n+1-\text { bits }} \overbrace{0 \ldots .00}^{2 n-\text { bits }} \bowtie \underbrace{d_{2,2 n-1} d_{2,2 n-2} \ldots d_{2,1} d_{2,0}}_{2 n-\text { bits }} \overbrace{0 \ldots .00}^{2 n+1-\text { bits }} \\
=\underbrace{x_{1,2 n} x_{1,2 n-1} \ldots x_{1,1} x_{1,0} d_{2,2 n-1} d_{2,2 n-2} \ldots d_{2,1} d_{2,0}}_{4 n+1} \\
=A_{4 n} A_{4 n-1} \ldots A_{1} A_{0}
\end{gathered}
$$

Whereas

$$
\begin{gathered}
L_{2}=-2^{2 n+1} d_{3} \\
=\underbrace{\bar{d}_{3,2 n-1} \bar{d}_{3,2 n-2} \ldots \bar{d}_{3,1} \bar{d}_{3,0}}_{L_{3}=-2^{2 n} d_{3}} \overbrace{11 \ldots 1}^{2 n+1}
\end{gathered}
$$

$$
\begin{gathered}
=\underbrace{\bar{d}_{3,2 n-1} \bar{d}_{3,2 n-2} \ldots \bar{d}_{3,1} \bar{d}_{3,0}}_{4 n} \overbrace{2 n-\text { bits }}^{2 n} \overbrace{11 \ldots 1}^{2 n} \\
L_{4}=2^{4 n+1} d_{3} \\
=\underbrace{d_{3,2 n-1} d_{3,2 n-2} \ldots d_{3,1} d_{3,0}}_{2 n-b i t s} \overbrace{00 \ldots 0}^{4 n+1}
\end{gathered}
$$

The schematic diagram for the reverse process is shown in 
Figure 4. The anticipated implementation of the scheme is based on simple CSAs and CPAs. It begins with an operands preparation unit (OPU) which prepares and manipulates the routing of the bits of the respective residues once inputted. The second MRD, $d_{2}$ is computed using CPA1 which is $2 n$ bits wide; the concatenation of bits does not require a hardware unit, but its results is relevant for further processing. CSA1 and CPA2 are used to compute for the third MRD, $d_{3}$ after which, the rest of the addition process is done in a cascading fashion thereby reducing the complexity which results in a simplified architecture. CPA 3 computes the save and carry from CSA 5 in order to get back the decimal or binary number, $X$. Regarding the hardware requirements of the unit; the total hardware (area) requirement is $(30 n+5)$ bits of full adders and a delay imposition of $(10 n+6)$ bits of full adders.

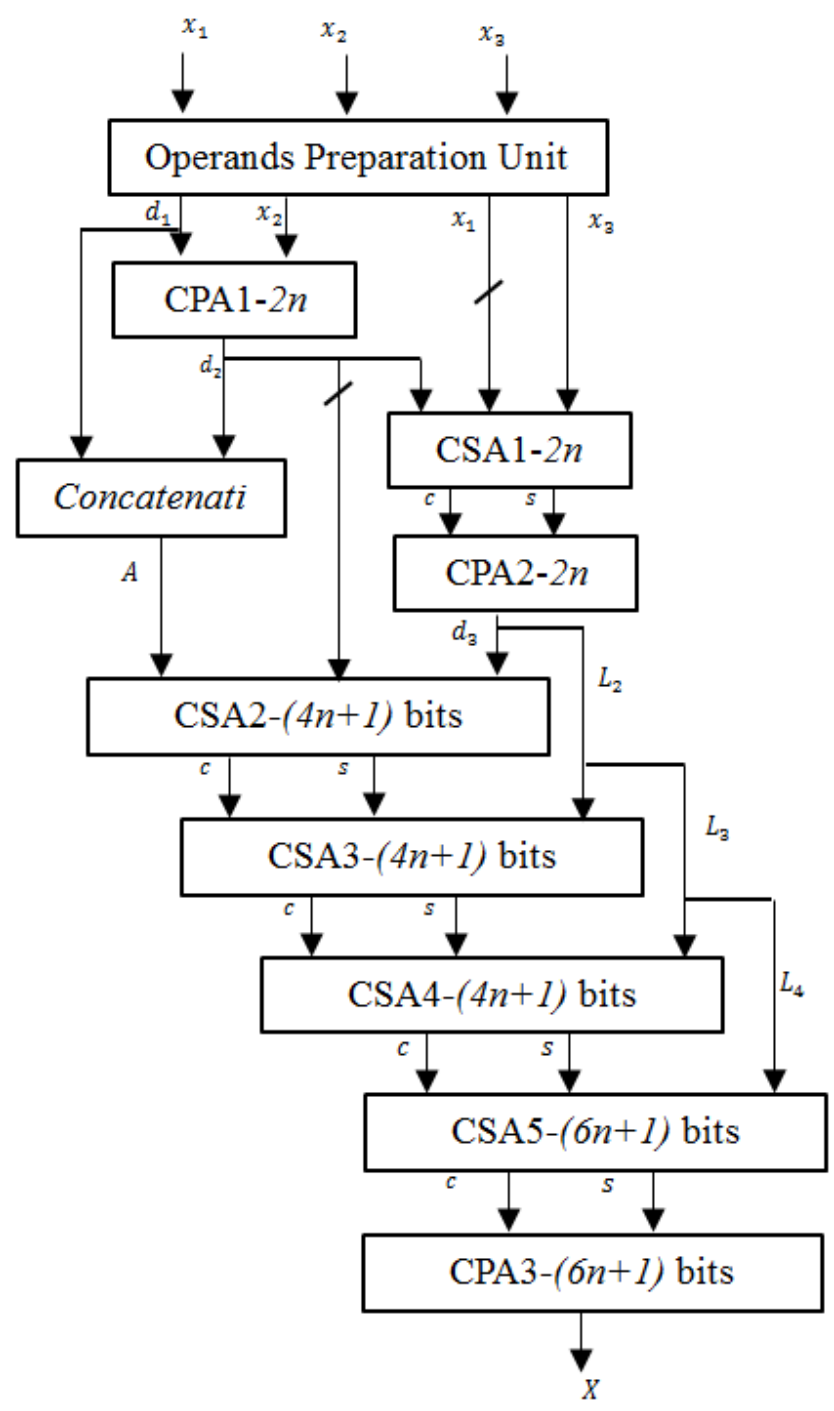

Figure 4. Schematic Diagram for Reverse Conversion Process.

\section{Numerical Illustration}

Given the moduli set $\left\{2^{2 n+1}-1,2^{2 n}-1,2^{2 n}\right\}$, take $n=2$. Consider $X$ representing a character of a message to be transmitted, assume the ASCII value for that message $X=$
6892. Then the conversion process is as follows; $6892=$ 1101011101100 (13-bits, since $X$ is a $(6 n+1)$-bit number) Thus, $\Lambda_{1}=1100, \Lambda_{2}=1110$ and $\Lambda_{3}=11010$

Therefore;

$$
\begin{gathered}
|6892|_{2^{5}-1}=|6892|_{31}=\left.|12+| 16(14)\right|_{31}+\left.|8(26)|_{31}\right|_{31} \\
=|12+7+22|_{31}=|41|_{31}=10 \\
|6892|_{2^{4}-1}=|6892|_{15}=\left.\left.|12+14+| 26\right|_{15}\right|_{15} \\
=|12+14+11|_{15}=|37|_{15}=7
\end{gathered}
$$

And $|6892|_{2^{4}}=|6892|_{16}=12$

And so $|6892|_{31|15| 16}=(10,7,12)_{31|15| 16}$

$$
10=010107=011112=1100
$$

And so $|6892|_{31|15| 16}=(10,7,12)_{31|15| 16}$

From the above illustration, it can be seen that the traditional method will convert the ASCII message, $\mathrm{X}=$ 6892 , to binary which is $6892=1101011101100$ (13-bits, since $X$ is a $(6 n+1)$-bit number). But this representation has a unique equivalent in RNS which is $|6892|_{31|15| 16}=$ $(10,7,12)_{31|15| 16}$. Each of the moduli sets are channels that can be used to transmit the message $\mathrm{X}$, but it is the binary equivalent of the residues that will be transmitted. The binary equivalent of $(10,7,12)_{31|15| 16}$ is as follows:

$$
10=010107=011112=1100
$$

In order to perform the reverse conversion we find the MRD's $a_{1}, a_{2}$ and $a_{3}$ and compute the MRC in order to get back the value of $X$ (i.e. message transmitted):

But $a_{1}=x_{1}=10$

$$
\begin{gathered}
a_{2}=|7-10|_{15}=12 \\
a_{3}=|((12-10) \times 5-12) \times 15|_{16}=14
\end{gathered}
$$

Therefore,

$$
X=10+12(31)+14(31)(15)=6892
$$

\section{Performance Analysis}

The performance of the communication link is determined by the energy per bit value. We can compare the worst case scenario of RNS architecture with the traditional method in terms of computing the bit energy. Let's assume a carrier power of $1 \mathrm{~W}$ at the transmitter, with the traditional method, the transmit energy per bit, $e_{b}=\frac{1}{13}=0.07692307692$ joules. In RNS representation, in the worst case scenario, $e_{b}=\frac{1}{5}=0.2$ joules. It can be seen that excess energy of 0.1230769231 joules is available in RNS than in the traditional method. The RNS architecture therefore will perform better than the traditional method in the unlikely event of rainfall along the satellite communication path. In the second and third transmission channels, $e_{b}=\frac{1}{4}=$ 0.25 joules. Table 1 shows the variation in the energy per bit 
between the proposed scheme using RNS and the traditional method. From Figure 5 it can be seen that for all values of $\mathrm{n}>1$, the proposed scheme will always outperform the traditional method. Therefore when there is rain fade along the satellite link, all other things being equal the proposed scheme will have enough energy available at the receiver to allow for proper data decoding at an acceptable bit error rate. It is also interesting to note that even the energy difference between the proposed scheme and the traditional scheme is higher than that of the traditional scheme. This can be seen in Figure 6.

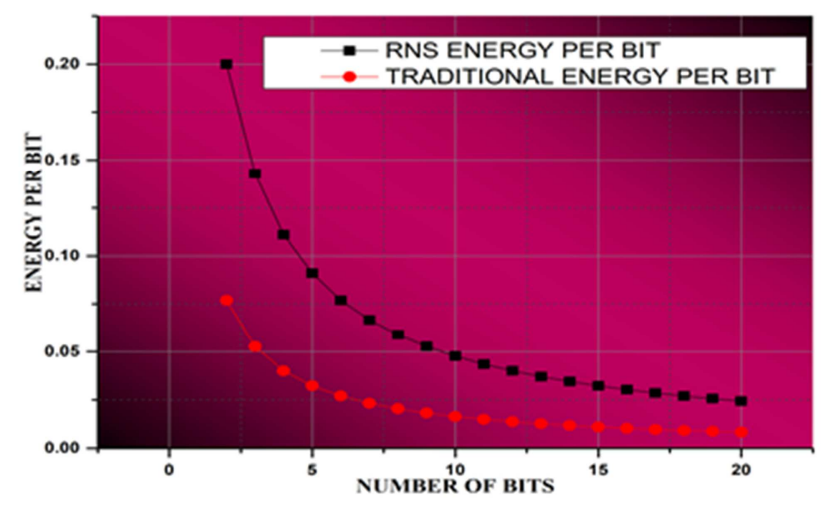

Figure 5. Variation in Energy per Bit.

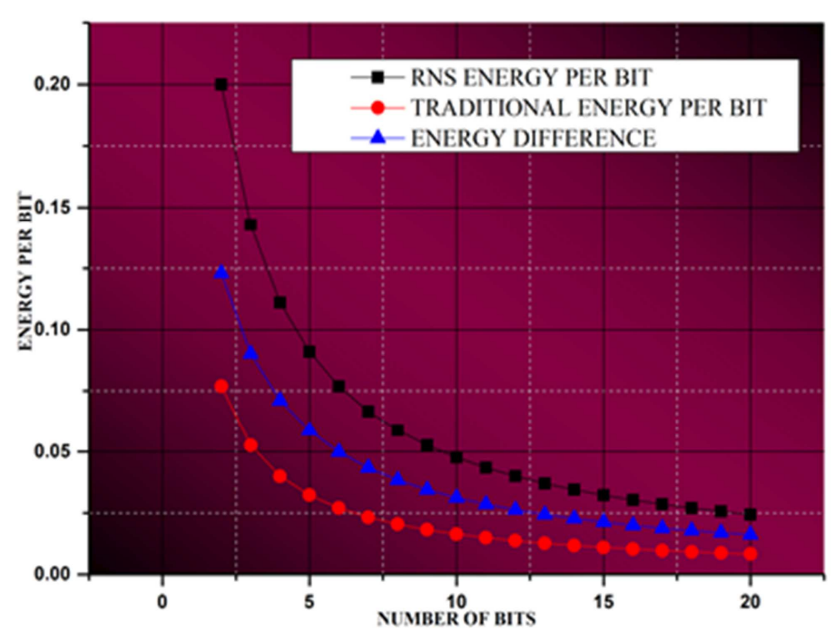

Figure 6. Difference in Energy per Bit.

Table 1. Variation in Energy per Bit.

\begin{tabular}{llll}
\hline $\mathbf{N}$ & Rns energy per bit & $\begin{array}{l}\text { Traditional Energy } \\
\text { Per Bit }\end{array}$ & Energy difference \\
\hline 2 & 0.2 & 0.076923077 & 0.123076923 \\
3 & 0.142857143 & 0.052631579 & 0.090225564 \\
4 & 0.111111111 & 0.04 & 0.071111111 \\
5 & 0.090909091 & 0.032258065 & 0.058651026 \\
6 & 0.076923077 & 0.027027027 & 0.04989605 \\
7 & 0.066666667 & 0.023255814 & 0.043410853 \\
8 & 0.058823529 & 0.020408163 & 0.038415366 \\
9 & 0.052631579 & 0.018181818 & 0.034449761 \\
10 & 0.047619048 & 0.016393443 & 0.031225605 \\
11 & 0.043478261 & 0.014925373 & 0.028552888 \\
12 & 0.04 & 0.01369863 & 0.02630137 \\
13 & 0.037037037 & 0.012658228 & 0.024378809 \\
14 & 0.034482759 & 0.011764706 & 0.022718053 \\
15 & 0.032258065 & 0.010989011 & 0.021269054 \\
\hline
\end{tabular}

\begin{tabular}{llll}
\hline $\mathbf{N}$ & Rns energy per bit & $\begin{array}{l}\text { Traditional Energy } \\
\text { Per Bit }\end{array}$ & Energy difference \\
\hline 16 & 0.03030303 & 0.010309278 & 0.019993752 \\
17 & 0.028571429 & 0.009708738 & 0.018862691 \\
18 & 0.027027027 & 0.009174312 & 0.017852715 \\
19 & 0.025641026 & 0.008695652 & 0.016945373 \\
20 & 0.024390244 & 0.008264463 & 0.016125781 \\
\hline
\end{tabular}

\section{Conclusion and Future Work}

A novel rain attenuation mitigation technique has been proposed using RNS architecture on the satellite communication link. This solution does not require human intervention unlike other mitigation techniques. The technique involves the use of converters and a different number system from the traditional number system to reduce the number of bits to be transmitted. Forward converters to convert to RNS system before transmission and reverse converters to convert back to the traditional number system at the receiver. The moduli set chosen, $\left\{2^{2 n+1}-1,2^{2 n}-1,2^{2 n}\right\}$, works for both odd and even numbers of $n$. Therefore, if the hardware realization of the proposed architecture is incorporated into the satellite communication system, it will help mitigate the attenuation due to rain since more energy will be made available to the individual bits. In future, the proposed scheme will be expanded to include a mechanism to detect and correct errors during transmission.

\section{Conflict of Interest}

The authors declare no conflict of interest in this research work.

\section{References}

[1] Crane, R. (1997). Electromagnetic Wave Propagation through Rain, John Wiley, New York, 1996, Chapter 2-3.

[2] Ulaganathen K., Rahman A. T., Islam R. and Malek A. N (2015), Mitigation Technique for Rain Fade Using Frequency Diversity Method, IEEE $12^{\text {th }}$ Malaysia International Conference on Communications (MICC), Kuching, Malaysia, November 2015.

[3] Nwaogu C. C., Amadi A. O., and Alozie I. S (2019), Mitigating Rain Attenuation on Wireless Communication Link Using Adaptive Power Control, Proceedings of the World Congress on Engineering and Computer Science WCECS 2019, San Francisco, USA, pp. 22-24.

[4] Ippolito J. Louis (1986), Radiowave Propagation in Satellite Communications, Van Nostrand Reinhold Company, New York.

[5] Patel, B. (2007). Emerging Digital Transmission Techniques for HDTV.

[6] Claudio, J., R., and Jacob, S. (2003). Adaptive Image Denoising and Edge Enhancement I Scale-Space using Wavelet Transforms. Pattern Recognition Letters, Volume 13, No. 7, pp 965-971. 
[7] Taylor F. J. (1984): "Residue Arithmetic: A Tutorial with Examples," Computer, vol. 17, no. 5, pp. 50-62.

[8] Arash H., Keivan N. and Reza R.(2008): A new high dynamic range moduli set with efficient reverse converter, $A n$ internation Journal of Computers and Mathematics with Applications Vol 55, Issue 4,, pp. 660-668.

[9] Gbolagade, K., A. (2010). Effective Reverse Conversion in Residue Number System Processors. PhD thesis, Delft University of Technology (TU-Delft), The Netherlands.

[10] Molahosseini A. S. and Navi K. (2007), New Arithmetic Residue to Binary Converters, International Journal of Computer Sciences and Engineering Systems, Vol. 1, No. 4, pp. 296-300.

[11] Szabo N. S. and Tanaka R. I. (1967): Residue Arithmetic and its Applications to computer Technology, New York: McGrawHill.

[12] Soderstrand M. A., Jenkins W. K., Jullien G. A. and Taylor F. J. (1986): Residue Number System Arithmetic: Modern Applications in Digital Signal Processing New York: IEEE Press.
[13] Wang Y., Xiaoyu S., and Mostapha A. (2002). Adder Based Residue to Binary Number Converters, IEEE Transactions On Signal Processing, Vol. 50, No. 7, pp. 1772-1779.

[14] Haron, N., Z. and Hamdioui, S. (2009). Residue-Based Code for Reliable Hybrid Memories. Proceedings of IEEE/ACM International Symposium on Nanoscale Architecture (NanoArch), pp. 27-32.

[15] Cao B., Chip-Hong C. and Thambipillai S. (2003): Adder Based Residue to Binary Converters for a New Balanced 4Moduli Set, Proceedings of the $3^{\text {rd }}$ International Symposium on Image and Signal Processing and Analysis, vol. 2 pp. 820825.

[16] Gbolagade K. A. (2013). An Efficient MRC based RNS-toBinary Converter for the, $\left\{2^{2 n+1}-1,2^{\mathrm{n}}, 2^{2 \mathrm{n}}-1\right\}$ Moduli Set, International Journal of Advanced Research in Computer Engineering \& Technology, (IJARCET), vol. 2, issue $10 \mathrm{pp}$ : 2661-2664.

[17] Siewobr H. and Gbolagade K. A. (2014). Modulo Operation Free Reverse Conversion in the Moduli Set $\left\{2^{2 n+1}-1,2^{n}, 2^{2 n}-1\right\}$ International Journal of Computer Applications (0975 8887) Volume 85-No 18. 